\title{
Adaptive Fractional Image Enhancement Algorithm Based on Rough Set and Particle Swarm Optimization
}

\author{
Xuefeng Zhang ${ }^{1, *,+} \mathbb{D}, \operatorname{Ri~Liu}^{2, \dagger}$, Jianxu Ren ${ }^{2,+}$ and Qinglong Gui ${ }^{2,+}$ \\ 1 College of Sciences, Northeastern University, Shenyang 110819, China \\ 2 School of Mechanical Engineering and Automation, Northeastern University, Shenyang 110819, China; \\ 1r1612281433@163.com (R.L.); r2646629596@163.com (J.R.); g19983972782@163.com (Q.G.) \\ * Correspondence: zhangxuefeng@mail.neu.edu.cn \\ + These authors contributed equally to this work.
}

check for updates

Citation: Zhang, X.; Liu R.; Ren J.; Gui, Q. Adaptive Fractional Image Enhancement Algorithm Based on Rough Set and Particle Swarm Optimization. Fractal Fract. 2022, 6, 100. https://doi.org/10.3390/ fractalfract6020100

Academic Editor: Norbert Herencsar

Received: 12 January 2022

Accepted: 3 February 2022

Published: 11 February 2022

Publisher's Note: MDPI stays neutral with regard to jurisdictional claims in published maps and institutional affiliations.

Copyright: (C) 2022 by the authors. Licensee MDPI, Basel, Switzerland. This article is an open access article distributed under the terms and conditions of the Creative Commons Attribution (CC BY) license (https:// creativecommons.org/licenses/by/ $4.0 /)$.
Abstract: This paper proposes a new image enhancement algorithm. At first, the paper uses the combination of rough set and particle swarm optimization (PSO) algorithm to distinguish the smooth area, edge and texture area of the image. Then, according to the results of image segmentation, an adaptive fractional differential filter is used to enhance the image. Finally, the experimental results show that the image enhanced by this algorithm has clear edge, rich texture details, and retains the information of the smooth area of the image.

Keywords: image enhancement; fractional differential; rough set; particle swarm optimization; adaptive algorithm

\section{Introduction}

Image enhancement is to improve the visual effect of the image. It is also an important issue in many fields such as pattern recognition, robotics, medical image processing [1-3], and remote sensing [4-7]. There are two types of image enhancement methods, one is the frequency domain method and the other is the spatial domain method [8-10]. Based on twodimensional Fourier transform, frequency domain method performs signal enhancement. Spatial domain method directly performs calculations on the gray level of the image when processing the algorithm based on the spatial domain. In the past, image enhancement used integer orders differential operator to enhance the image, but the effect is not ideal, there are problems such as insignificant enhancement and destruction of smooth areas. Therefore, it is necessary to improve the traditional image enhancement algorithm to improve the effect of enhanced image.

In the past few years, fractional differential operator has gradually been used to process images [11-13], and ref. [14] used the fractional order defined by Grünwald-Letnikov to design a fractional differential mask, which introduces the fractional order into image processing. Ref. [15] proposed an adaptive fractional total variation image restoration method, and ref. [16] proposed a multi-scale denoising model based on fractional partial differential equations. At present, the use of fractional differential operators to process images has become a hot issue. Many references have applied this method and achieved certain results. Ref. [17] introduced a one-dimensional Savitzky-Golay filter, extended it from integer order to fractional order, and from one dimension to two dimensions. Then, proposed an image enhancement algorithm based on two-dimensional Savitzky-Golay filter, and got the smooth area of the image has hardly changed, and the edge details have also been enhanced, but the enhancement effect is not outstanding. The introduction of fractional methods in the field of image processing has remained popular in recent years. Ref. [18] proposed the AFDA (Adaptive Fractional Differential Algorithm) algorithm is used to enhance medical images, but its algorithm will cause excessive enhancement in 
local areas. Ref. [19] proposed an image segmentation and enhancement algorithm, but the disadvantage is lower image contrast.

Rough set and PSO are also used in the image processing. Rough set was first proposed by Polish professor Z. Pawlak in 1982. It is another mathematical method for processing uncertain information after probability theory and fuzzy set theory $[20,21]$. As a mathematical tool for describing uncertainty, it can effectually analyze and process incomplete information such as inconsistency, inaccuracy and incompleteness, it can find hidden knowledge and reveal potential laws [22,23]. Ref. [23] combined rough set and kernel PCA method, proposed a 3D MR image denoising algorithm, ref. [24] proposed a robust modified Gaussian mixture model with rough set for image segmentation. Ref. [25] proposed a soft fuzzy rough set-based MR brain image segmentation.

PSO is an evolutionary form research on bird predation behavior [26]. Finding the optimal solution which is shared among individuals in the group through collaboration and information is the basic idea of PSO. The advantage of PSO is that it is simple to implement and does not have many parameter adjustments. Ref. [27] combined integrating fuzzy entropy clustering with an improved particle swarm optimization, proposed a new clustering method for segmentation of Magnetic resonance imaging (MRI) brain images. Ref. [28] proposed a color image segmentation based on multi-level Tsallis-Havrda-Charvát entropy and 2D histogram using PSO algorithms.

Based on these previous works, in order to enhance the texture area and edge of the image while retaining the characteristics of the smooth area, an adaptive fractional-order image enhancement algorithm based on image segmentation is proposed. To achieve the desired purpose, the main difficulty lies in accurately segmenting the texture area, edge and smooth area of the image and how to find the suitable fractional order of image enhancement. The main contributions of the paper are summarized as follows:

- An image segmentation method based on rough set and PSO is proposed, which accurately segments the image into smooth area, edge and texture area to prepare for the next step of image enhancement.

- According to the local feature information of the image, an adaptive image enhancement algorithm is designed by using fractional differential filter.

- The image is enhanced by combining the results of image segmentation, so that the edge and texture area of the image are effectively enhanced while the characteristics of the smooth area of the image are well preserved.

- Compared with the refs. $[14,17,19]$, the advantage of the algorithm in the paper is that it combines the rough set and PSO methods to accurately segment the image, and carries out targeted enhancement according to the segmentation results. The adaptive fractional enhancement algorithm proposed in the paper enhances the edge details of the image more obviously, and the texture details are more prominent, which better overcomes the shortcomings of the traditional fractional filter enhancement that will make the image blurry and the enhancement effect is not obvious.

The rest structure of the paper are as follows. Section 2 introduces the related knowledge of rough set, PSO and fractional differential filter. Section 3 proposes image segmentation methods based on rough set and particle swarm optimization, and an adaptive image enhancement algorithm is proposed. The experimental results are given in Section 4 to illustrate the effectiveness of the algorithm. Finally, the conclusions are given in Section 5 .

\section{Preliminaries}

\subsection{Rough Set}

Generally, the whole of the research object is called the domain of discourse $U$, and $x_{i}$ is the element in $U$, that is, the decision object. A part of $U$ is called a subset. If $X_{i}(i \leq k)$ is a subset of $U$, and $X_{i} \neq \varnothing(i \leq k), X_{i} \cap X_{j}=\varnothing(i \neq j), \bigcup_{i=1}^{k} X_{i}=U$, then $\left\{X_{i} \mid i \leq k\right\}$ is called the division of the universe of $U$, that is, the research objects are divided into different categories. Let $R \subseteq U \otimes U$, call $R$ the relationship on $U$. If the relationship $R$ on $U$ satisfies: 
(1) Reflexivity: $\left(x_{i}, x_{i}\right) \in R\left(x_{i} \in U\right)$;

(2) Symmetry: if $\left(x_{i}, x_{j}\right) \in R$, then $\left(x_{j}, x_{i}\right) \in R,\left(x_{i}, x_{j} \in U\right)$;

(3) Transitivity: if $\left(x_{i}, x_{j}\right) \in R,\left(x_{j}, x_{k}\right) \in R$, then $\left(x_{i}, x_{k}\right) \in R\left(x_{i}, x_{j}, x_{k} \in U\right)$.

Then, $R$ is called the equivalent relationship on $U$, and the equivalent relationship is also called the indistinguishable relationship. Using the equivalent relationship to classify the universe, using $\left[x_{i}\right]_{R}$ represents the set of all objects that are indistinguishable from $x_{i}$ under the equivalence relation $R$, that is, the equivalent of the element $x_{i}$ under $R$, then $\left[x_{i}\right]_{R}$ can be expressed as,

$$
\left[x_{i}\right]_{R}=\left\{x_{j} \mid\left(x_{i}, x_{j}\right) \in R\right\}\left(x_{i}, x_{j} \in U\right) .
$$

Regarding the information system $K=(U, R)$ as an approximate space, $R$ is the equivalence relation on the universe $U, x_{i}$ is an object in $\mathrm{U},\left[x_{i}\right]_{R}$ represents a collection of all objects which are indistinguishable from $x_{i}$, that is, the equivalence class of $R$. When a collection $\left[x_{i}\right]_{R}$ can be accurately described by the attributes of $R,\left[x_{i}\right]_{R}$ is considered to be defined by $R$ and is called a definable or exact set of $R$; when $\left[x_{i}\right]_{R}$ cannot be accurately described by the attributes of $R,\left[x_{i}\right]_{R}$ is an undefined set or rough set of $R$.

In rough set method, the approximate definition of set $X$ about $R$ is,

$$
\underline{R}(x)=\left\{x_{i} \mid x_{j} \in U,\left[x_{i}\right]_{R} \subseteq X\right\} .
$$

The upper approximation of the set $X$ on $R$ is defined as,

$$
\bar{R}(x)=\left\{x_{i} \mid x_{i} \in U,\left[x_{i}\right]_{R} \cap X \neq \varnothing\right\} .
$$

The boundary area of the set $X$ on $R$ is defined as,

$$
B N_{R}(X)=\bar{R}(x)-\underline{R}(x) .
$$

The upper approximation set $\bar{R}(x)$ of set $X$, lower approximation set $\underline{R}(x)$ and boundary $B N_{R}(X)$ as shown in the Figure 1.
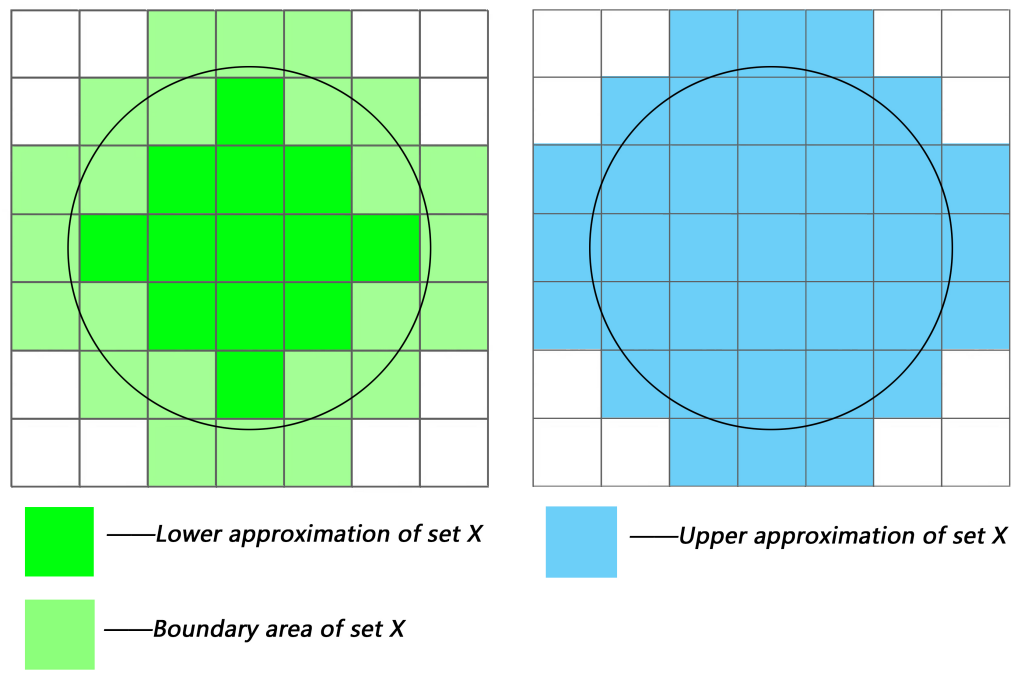

Figure 1. Rough set schematic.

The more elements the boundary set $B N_{R}(X)$ contains, the set $X$ are more uncertain. The uncertainty of the set $X$ is roughness, which is defined as follows,

$$
\rho_{R}(X)=1-\frac{|\underline{R}(X)|}{|\bar{R}(X)|} .
$$


The roughness function reflects the uncertainty of the set $X$. The larger the roughness value, the greater the uncertainty of the set $X$.

\subsection{Particle Swarm Optimization}

Particle swarm optimization is inspired by the behavioral characteristics of bird populations to solve optimization problems, and proposed by Kennedy and Eberhart in 1995 [26]. The particle swarm algorithm first initializes a group of particles that may represent the optimal solution in the solvable space, and uses velocity, position and fitness value to represent the characteristics of each particle. Among them, the fitness value is calculated by the fitness value function, and its value represents the quality of the particle. The optimal position of the fitness value calculated from the positions experienced by the particle is called the individual extremum, and the optimal position searched by all particles becomes the group extremum. The particle continuously updates its speed and position by tracking the individual extremum and the group extremum. Every time the particle updates its position, the fitness value is calculated once, and by comparing the fitness value of the new particle with the individual extremum to update the individual extremum and position of the group extremum. Until the end of the iteration, find the optimal solution in the solution space.

Supposing there are $n$ particles in a D-dimensional space, where the position of the ith particle is denoted by $x_{i D}$ and the velocity is denoted by $V_{i D}$. The corresponding individual extremum is denoted as $p_{i D}$, and the group extremum is denoted as $g_{i D}$. In each iteration process, the particle updates its speed and position through the individual extremum and the global extremum. The update formula Equations (6) and (7) is as follows:

$$
\begin{gathered}
V_{i d}^{t+1}=V_{i d}^{t}+c_{1} \cdot r_{1}\left(p_{i d}-x_{i d}^{t}\right)+c_{2} \cdot r_{2}\left(g_{i d}-x_{i d}^{t}\right)(i=1,2, \cdots, m ; d=1,2, \cdots, D), \\
x_{i d}^{t+1}=x_{i d}^{t}+V_{i d}^{t+1}(i=1,2, \cdots, m ; d=1,2, \cdots, D),
\end{gathered}
$$

among them, generally $V_{i} \in\left[-V_{\max }, V_{\max }\right], V_{\max }$ is specified by the user; $V_{i d}^{t}$ is the velocity of the i-th particle in the D-dimensional space after the $\mathrm{t}$-th iteration; $x_{i d}^{t}$ is the location of the $\mathrm{i}$-th particle in the $\mathrm{D}$-dimensional space after the $\mathrm{t}$-th iteration, $c_{1}$ and $c_{2}$ are non-negative constants called acceleration factors, and $r_{1}$ and $r_{2}$ are random numbers distributed between $(0,1)$. The acceleration factor has the ability to make the particles approach the extremum position of the group, find the optimal solution in space, and prevent the particles from blindly searching.

\subsection{Enhancement Effect of Fractional Differential on Signal}

The definition of fractional order has been proposed for more than 300 years ago, and it has developed rapidly in recent years [29-32]. So far, there are three main forms of fractional definition expressions: Riemann-Liouville definition, Caputo definition and Grünwald-Letnikov defifinition [33]. The Riemann-Liouville definition is as follows,

$$
{ }_{a}^{R} D_{t}^{\alpha}=\frac{1}{\Gamma(n-\alpha)}\left(\frac{d}{d t}\right)^{n} \int_{a}^{t} \frac{f(\tau)}{(t-\tau)^{\alpha-n+1}} d \tau,
$$

where $n-1<\alpha<n, \Gamma(\cdot)$ is the Gamma function. The Caputo definition is as follows,

$$
{ }_{a}^{C} D_{t}^{\alpha}=\frac{1}{\Gamma(n-\alpha)} \int_{a}^{t} \frac{f^{(n)}(\tau)}{(t-\tau)^{\alpha-n+1}} d \tau .
$$

The above two definitions both use the Cauchy integral formula, which is relatively complicated and is not conducive to large-scale data calculations. The Grünwald-Letnikov, referred definition can be transformed into a convolutional form, so it is compared with the other two definitions have better effects in image processing. The Grünwald-Letnikov definition of function $f(t)$ is as follows, 


$$
{ }_{a}^{G} D_{t}^{\alpha}=\lim _{h \rightarrow 0} h^{-\alpha} \sum_{j=0}^{\left[\frac{t-a}{h}\right]}(-1)^{j}\left(\begin{array}{c}
\alpha \\
j
\end{array}\right) f(t-j h),
$$

in the Equation (10), $\left(\begin{array}{c}\alpha \\ j\end{array}\right)$ represents the combination number parameter.

It is known that for any square integrable energy signal $f(t) \in L^{2}(R)$, its Fourier transform can be obtained from the theory of signal processing as,

$$
\hat{f}(w)=\int_{R} f(t) e^{-j w t} d t
$$

Suppose the n-th order derivative of the signal $f(t)$ is $f^{(n)}(t), n \in Z$, based on the properties of the Fourier transform,

$$
D^{n} f(t) \stackrel{F T}{\Leftrightarrow}(\hat{D} f)^{n}(w)=(j w)^{n} \cdot \hat{f}(w)=\widehat{d}^{n}(w) \widehat{f}(w) .
$$

If the fractional derivative of the v-order of signal $f(t)$ is $f^{(v)}(t), v \in R^{+}$, it can be obtained by the fractional Fourier transform [34],

$$
D^{v} f(t) \stackrel{F T}{\Leftrightarrow}(\widehat{D} f)^{v}(w)=\widehat{d}^{v}(w) \cdot \widehat{f}(w),
$$

where

$$
\left\{\begin{array}{l}
\widehat{d}^{v}(w)=(j w)^{v}=\widehat{\alpha}^{v}(w) \cdot d^{j \theta^{v}(w),} \\
\widehat{\alpha}^{v}(w)=|w|^{v}, \widehat{\theta}^{v}(w)=\frac{v \pi}{2} \operatorname{sgn}(w) .
\end{array}\right.
$$

From the above, the amplitude-frequency characteristic curves can be obtained, when takes the fractional order is $0.1,0.2,0.3,0.5,0.6,0,7,0.8,1.2$ as shown in Figure 2.

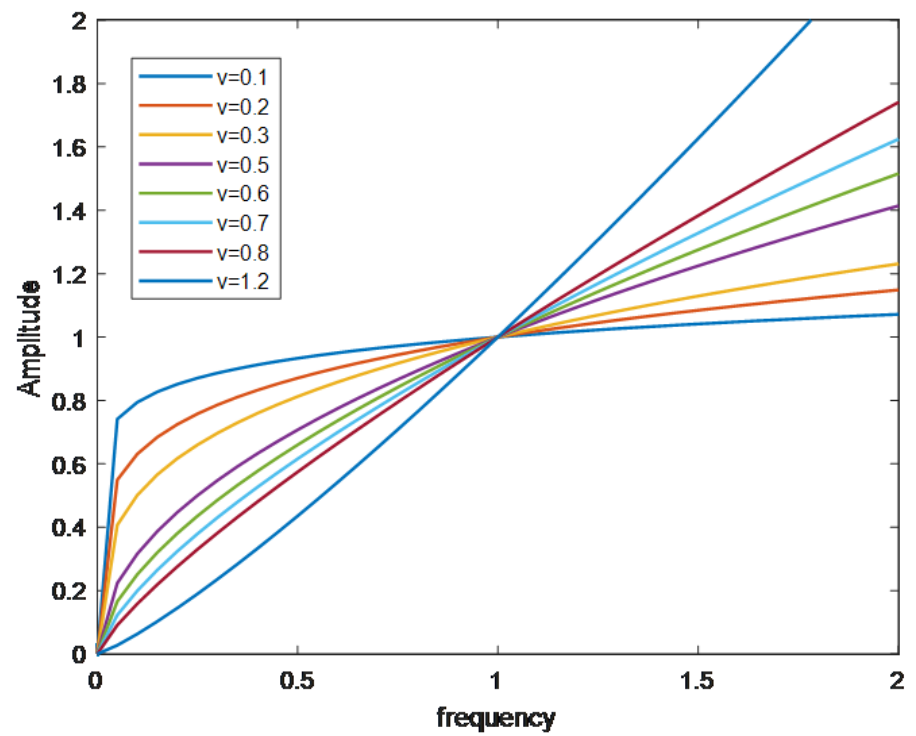

Figure 2. Amplitude frequency characteristic curve of fractional differential operator.

It can be clearly seen from Figure 2 that when the fractional order is below 0.5 , the corresponding differential operator has a certain increase in the signal amplitude, but the increase is relatively small compared to the fractional order above 0.5 . Therefore, the order of the fraction has different effects on the image. The differential order can be changed according to the local information and characteristics of the image, so as to achieve effective enhancement of different areas of the image. 


\section{Main Results}

The following is the main works of the paper. Firstly, an image segmentation algorithm is designed using the characteristics of rough set that can deal with incomplete information. According to the characteristic that PSO can find the optimal solution in a solution space, another image segmentation algorithm is proposed. Then, the advantages of these two segmentation algorithms are combined to segment the image. Finally, an adaptive fractional order algorithm is designed based on the local information characteristic of the image, and enhances the edge and texture areas of the image by the segmentation results.

The algorithm flow chart of the paper is as shown in Figure 3.

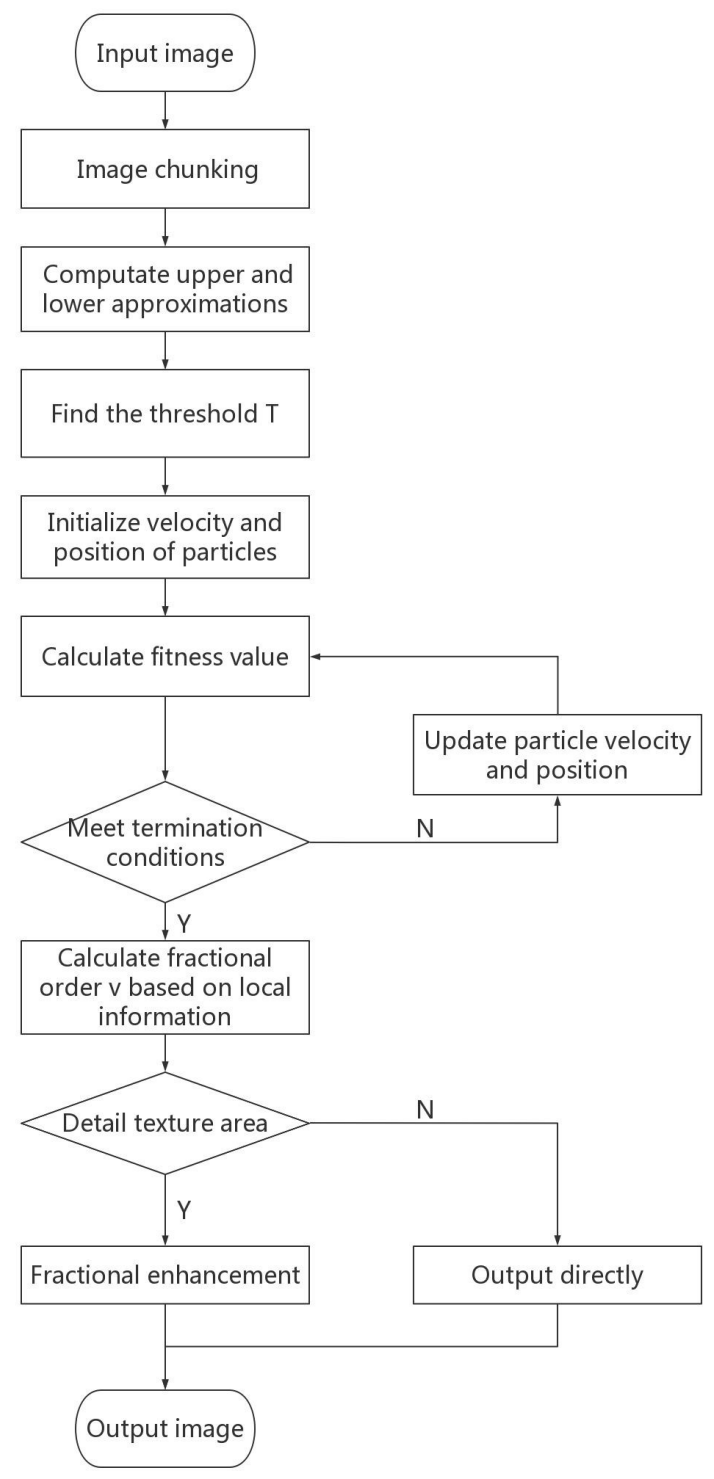

Figure 3. The algorithm flow chart of the paper.

\subsection{Image Segmentation Algorithm Based on Rough Set}

First of all, for the purpose of enhancing the image better, the smooth area, edge and texture regions of the image must be accurately segmented. An image segmentation method based on rough set is proposed here. Dividing the image into sub-blocks of the same size, with each sub-block as the smallest unit, the boundary threshold is calculated through the built-in function Otsu of matlab. Then, combining the rough set to find the best threshold for image segmentation. Experimental results show that this segmentation 
method effectively distinguishes the edge and texture regions of the image. The image segmentation algorithm steps based on rough set are as follows:

Step 1: For the image $I(i, j)$, the size of which is $m \times n$, dividing $I$ into $N$ sub-blocks according to the appropriate size, and mark the sub-blocks as $M_{t}(1 \leq t \leq N)$. Then, find the maximum gray value $M_{t \max }$ and the minimum gray value $M_{t \min }$ in each sub-block. Analyzing the results of many experiments, it is found that the more complex the image, the smaller the sub-block segmentation, the more accurate the segmentation. Therefore, the number of sub-blocks should be as suitable as possible for the image.

Step 2: Using the built-in function Otsu of matlab to calculate the optimal boundary threshold $T_{r}$ for each sub-block, and obtain the maximum and minimum thresholds $T_{\max }$ and $T_{\min }$. Combining rough set, calculate the upper and lower approximate values of the target $\bar{O}_{\tau}, \underline{O}_{\tau}$ and the background $\bar{B}_{\tau}, \underline{B}_{\tau}$ by Equations (15)-(18):

$$
\begin{aligned}
& \bar{O}_{\tau}=\left\{M_{t} \mid M_{t \min } \leq M(i, j) \leq T_{\max }, r \in[1, N]\right\}, \\
& \underline{O}_{\tau}=\left\{M_{t} \mid M_{t \max } \leq M(i, j) \leq T_{\max }, r \in[1, N]\right\}, \\
& \bar{B}_{\tau}=\left\{M_{t} \mid T_{\min } \leq M(i, j) \leq M_{t \text { max }}, r \in[1, N]\right\}, \\
& \underline{B}_{\tau}=\left\{M_{t} \mid T_{\min } \leq M(i, j) \leq M_{t \min }, r \in[1, N]\right\} .
\end{aligned}
$$

Step 3: After obtaining the upper and lower approximate values of the target and background, calculate the roughness and entropy by Equations (5) and (19). Then, find the maximum entropy corresponding to the pixel value of the image itself, which is the best threshold for segmenting the image. Using the best threshold to segment image I, and finally get the segmentation result.

$$
E=-\sum_{R=1}^{N} \rho_{R} \log \rho_{R},
$$

where $\rho_{R}$ is roughness of the R-th sub-block.

\subsection{Image Segmentation Algorithm Based on PSO}

It can be found from Figures $4 \mathrm{~b}$ and $5 \mathrm{~b}$ that the image segementation based on rough set has a ideal effect on the edge and texture area of the image, but the segmentation effect for the smooth area of the image is not obvious. Therefore, in order to better segment the smooth area of the image, an image segmentation method based on particle swarm optimization is proposed. At first, randomly selecting $\mathrm{n}$ particles, calculating the individual extremum of each particle to obtain the global extremum; then calculating the gray-scale fitness value of each particle, thereby updating the global extremum, the velocity and position of each particle. In the end of the cycle, the segmentation threshold $T$ is obtained. The defination of $T$ is as follows,

$$
T=\arg \max \left[H_{O}(T)+H_{B}(T)\right],
$$

where $H_{O}(T)$ and $H_{B}(T)$ represent the entropy of the target area and the background area in the image, respectively defined as:

$$
\begin{gathered}
H_{O}(T)=-\sum_{k}\left(P_{k} / P_{O}\right) \lg \left(P_{k} / P_{O}\right), k=0,1,2, \cdots, T-1 ; \\
H_{B}(T)=-\sum_{k}\left(P_{k} / P_{B}\right) \lg \left(P_{k} / P_{B}\right), k=T, T+1, T+2, \cdots, 255 ;
\end{gathered}
$$

where $P_{k}$ represents the number of pixels whose gray value is $k$. In an image, $P_{O}$ represents the total number of pixels whose gray value is lower than $T, P_{B}$ represents the total number of pixels in an image whose gray value is higher than $T$. 


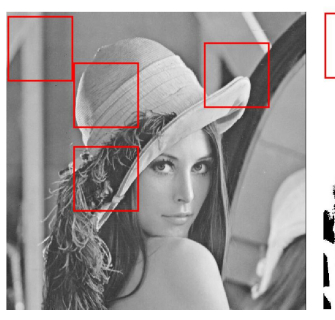

(a)

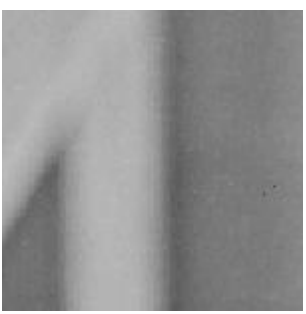

(e)

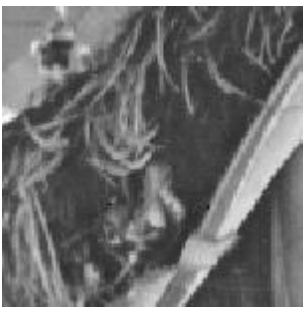

(i)

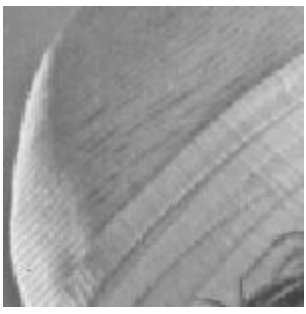

(m)

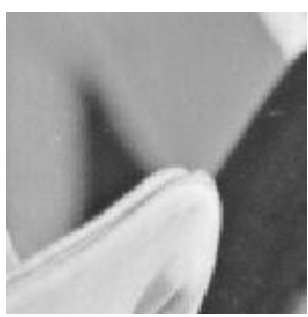

(q)

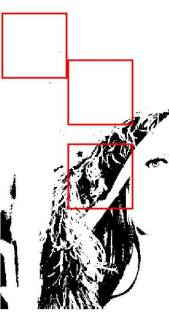

(b)

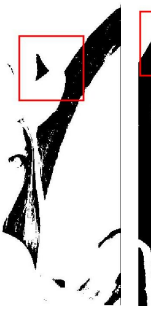

(f)

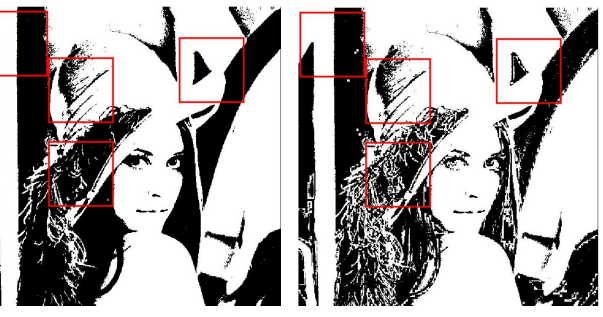

(c)

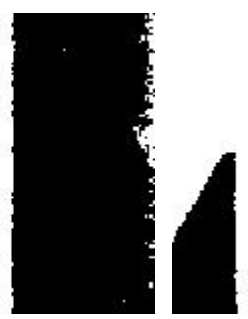

(d)

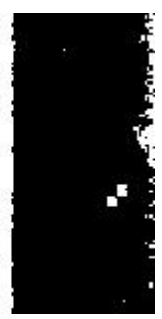

(h)

(g)

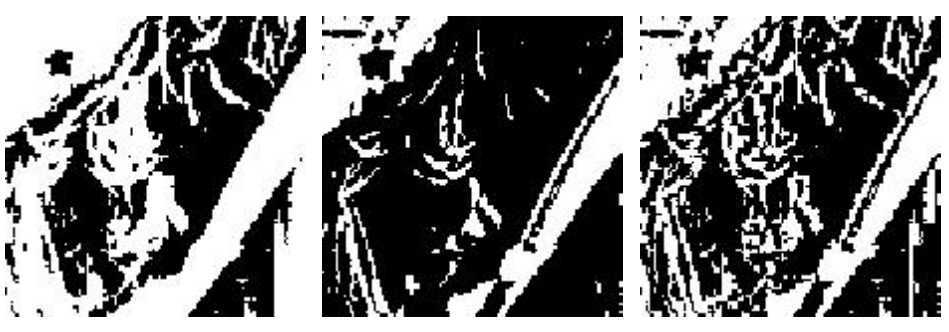

(j)

(k)

(1)

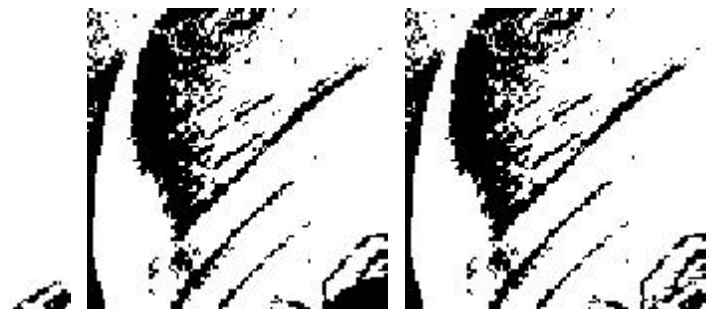

(o)

(p) $(\mathbf{n})$

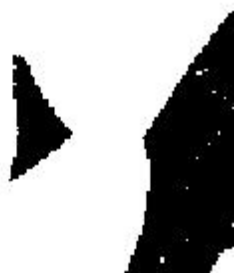

(r)

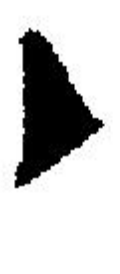

(s)
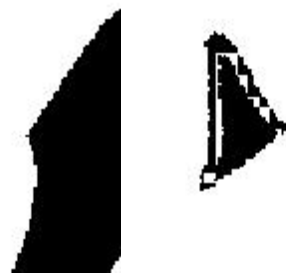

(t)

Figure 4. Lena image segmentation renderings and partial enlarged images under different algorithms. (a) is the original image of Lena, (b) is the segmentation effect of the rough set method, (c) is the segmentation effect of the PSO method, (d) is the combined segmentation effect of the two methods, $(\mathbf{e}-\mathbf{t})$ are the corresponding partial enlarged images. 


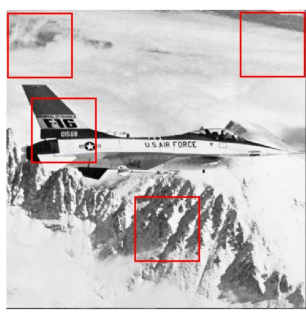

(a)

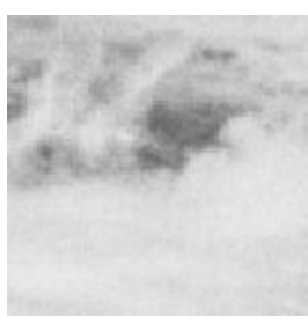

(e)

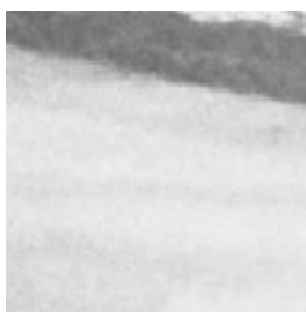

(i)

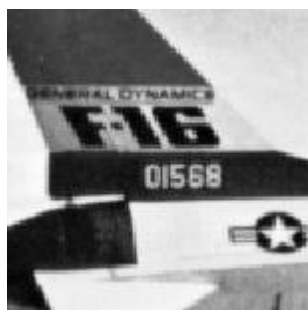

(m)

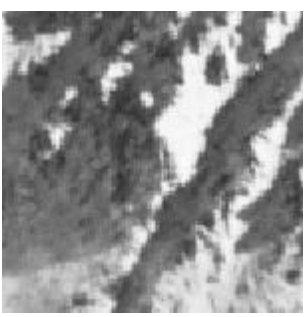

(q)

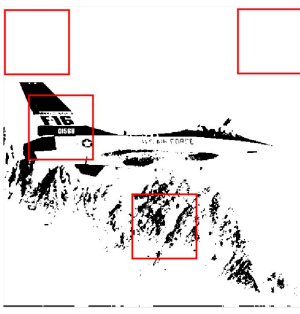

(b)

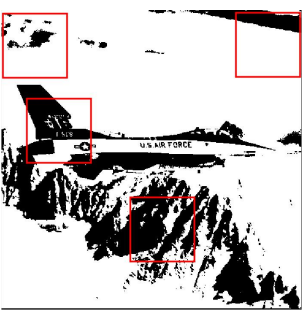

(c)

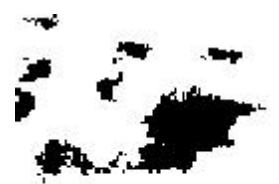

(g)
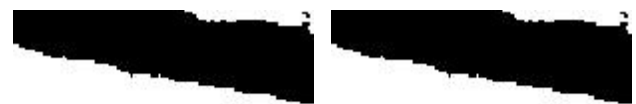

(k)

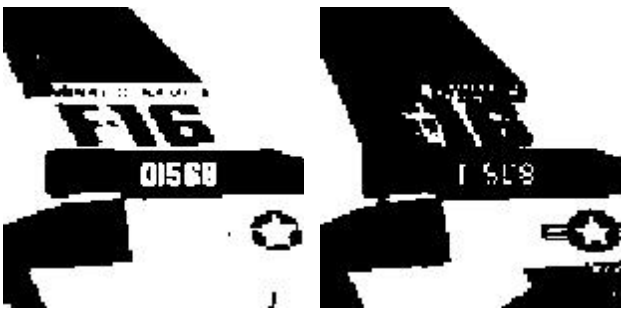

(n)

(o)

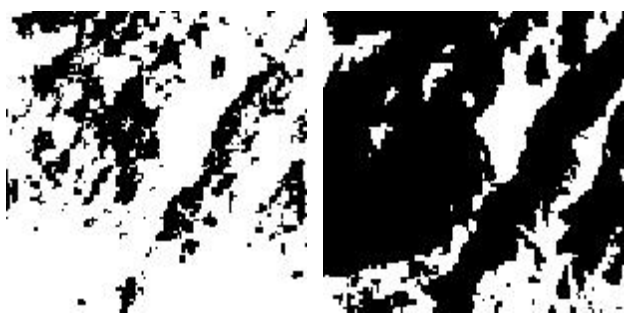

(s)

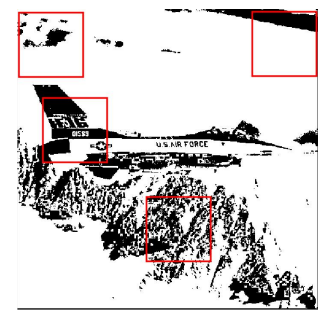

(d)

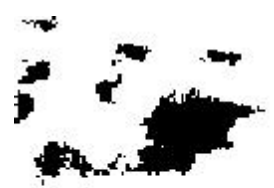

(h)

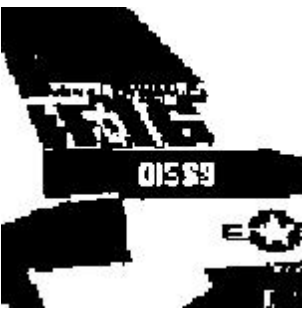

(p)

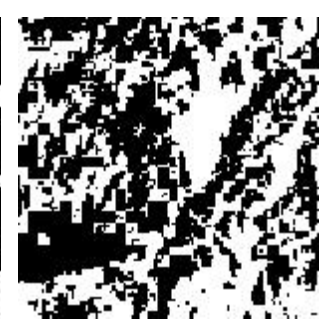

(t)

Figure 5. Plane image segmentation renderings and partial enlarged images under different algorithms. (a) is the original image of plane, (b) is the segmentation effect of the rough set method, (c) is the segmentation effect of the PSO method, (d) is the combined segmentation effect of the two methods, (e-t) are the corresponding partial enlarged images. 
The experimental results show that the use of PSO algorithm to perform secondary segmentation of the image makes the segmentation effect of the smooth area of the image more obvious. The steps of the image segmentation algorithm based on PSO are as follows:

Step 1: Randomly selecting p among the 256 gray values as the initial particle position ( $p=10$ in the paper), that is, the pbest position of each particle in the initial state, and randomly initializing the velocity $V_{i}\left(V_{i} \in[-20,20]\right)$ of each particle. Calculating the individual extreme value of each particle according to the Equation (20), the global extreme value is the largest of the individual extreme values, setting gbest as the current position of the particle.

Step 2: Calculating the gray scale fitness value of each particle according to the Equation (20). If it is better than the individual extreme value of the current position of the particle, set pbest to the position of the particle, record the position of the particle and update the global extreme value.

Step 3: Using Equations (6) and (7) to update the position and speed of each particle. When the particle gray value is greater than 255 , start counting from 0 , when the gray value is less than 0, counting backward from 255.

Step 4: In the end of cycle, getting the final gbest, that is to find the threshold $\mathrm{T}$ for image segmentation. The parameter is selected in the paper as $c 1=c 2=2$, which is obtained according to the experiments.

\subsection{Combination of Two Image Segmentation Methods}

According to the analysis in Figures $4 b, j$ and $5 b, n$, the rough set segmentation method has better segmentation effect in the texture area of the image, while the PSO method has better segmentation effect in the smooth area and edge of the image. Therefore, the paper combines these two methods to achieve accurate segmentation of image smooth area, edge and texture area. The combining steps in the paper are as follows:

Step 1: Dividing the two segmentation results into sub-blocks of moderate size, $G_{1}$ represents the number of pixels whose gray value is 0 in each two corresponding sub-blocks, and $G_{2}$ represents the number of pixels whose gray value is 255 in each two corresponding sub-blocks. If $G_{1}$ is less than half of the sum of the number of pixels in the two sub-blocks, it means that the area is more likely to have black background and white edge. If $G_{2}$ is less than half of the sum of the number of pixels in the two sub-blocks, it means that the area is more likely to have a black background and a white edge.

Step 2: Detecting sequentially the pixel values corresponding to the two segmentation results in turn. If the pixel values corresponding to the two segmentation results are both 0 , the pixel value of this point in the final segmentation image is 0 ; if both are 255, the pixel value of this point in the final segmentation image is 255; if the two are different, find what state the pixel belongs to in step 1 , if it is a white background and a black edge, the pixel value of this point is recorded as 0 ; if it is a black background and a white edge, the pixel value of this point is recorded as 255 .

\subsection{Adaptive Fractional Enhancement Algorithm}

Fractional differential filtering not only strengthens high-frequency signal of the image, but also retains some low-frequency information non-linearly. Therefore, in image processing, the fractional differential is used to enhance the edge texture details, and to a certain extent retain the information of the smooth area of the image.

For a two-dimensional image $g(s, t)$, the duration $[a, t]$ is equally divided by unit interval $l=1$, namely $n=\left[\frac{t-a}{l}\right]^{l=1}$. Then, the partial fractional differential of $g(s, t)$ in the $x$-direction and $y$-direction is:

$$
\begin{aligned}
& \frac{\partial^{v} g(s, t)}{\partial s^{v}} \approx g(s, t)+(-v) g(s-1, t)+\frac{(-v)(-v+1)}{2} g(s-2, t), \\
& \frac{\partial^{v} g(s, t)}{\partial t^{v}} \approx g(s, t)+(-v) g(s, t-1)+\frac{(-v)(-v+1)}{2} g(s, t-2) .
\end{aligned}
$$


Extend the above two Equations (23) and (24) to the other six directions, and get a fractional filter based on eight directions [14], as shown in Figure 6.

\begin{tabular}{|c|c|c|c|c|}
\hline$W_{3}$ & 0 & $W_{3}$ & 0 & $W_{3}$ \\
\hline 0 & $W_{2}$ & $W_{2}$ & $W_{2}$ & 0 \\
\hline$W_{3}$ & $W_{2}$ & $W_{1}$ & $W_{2}$ & $W_{3}$ \\
\hline 0 & $W_{2}$ & $W_{2}$ & $W_{2}$ & 0 \\
\hline$W_{3}$ & 0 & $W_{3}$ & 0 & $W_{3}$ \\
\hline
\end{tabular}

Figure 6. Franctional differencial filter.

$$
\left\{\begin{array}{l}
W_{1}=8 \times 1 \\
W_{2}=-v \\
W_{3}=\frac{-v(-v+1)}{2},
\end{array}\right.
$$

where $W_{1}, W_{2}, W_{3}$ can be calculated by the above formula, $v$ is the fractional order.

In the image enhancement process, the order of the fractional differential has a significant impact on the image enhancement effect. It is not only related to the average value of the gradient amplitude, the maximum of gradient amplitude and the minimum of gradient amplitude, but is also affected by the partial information of the image. The paper is to better enhance the edge details of image, an adaptive algorithm is designed according to the partial information of the image, which calculates the appropriate fractional order according to the partial information of the image.

Definition 1. [35,36]: Image entropy is characteristic statistical form that reflects the average amount of information in an image. Generally, the $S$ in the smooth area of the image is small and which of the edge detail area is large. The expression is as follows,

$$
S=-\sum_{I i j \in W} P_{i j} \log P_{i j}
$$

where $P_{i j}$ is the probability of pixel value $I_{i j}$ in the image.

Definition 2. [37]: For an image $G(x, y)$, suppose the average value of the gradient amplitude in 8 directions of each pixel in $G(x, y)$ is $K(i, j)$ and normalized. Taking the maximum of $K(i, j)$ as $X$, the minimum of $K(i, j)$ as $Y$, and dynamic amplitude expression $M(i, j)$ is obtained. The expression of the gradient magnitude $M$ is as follows,

$$
M=\frac{K-Y}{X-K}
$$

Definition 3. [38]: An image has image roughness $C$, this value represents the relative offset of the gray value of the image pixels, which is large in the texture region and small in the smooth region. The expression of $C$ is as follows,

$$
C=\frac{1}{1-\sigma^{4}}
$$

where $\sigma$ is the local mean square error of the image.

The three definitions mentioned above are image entropy, gradient magnitude and roughness. The common feature of these three definitions is that they are smaller in the smooth area of the image and larger in the texture area of the image, which means that the higher the value of the local information parameters $M, S, C$ are. If the value of $M, S, C$ are 
large, it indicates that the probability that the area is the target enhancement area is greater, and the fractional order should be larger; the smaller the value of $M, S, C$, the smaller the probability that the area is the target enhancement area, and the fractional order should also be smaller. It can be seen that the size of the fractional order is positively correlated with the size of the local parameter values of the three images.

$k_{1}, k_{2}, k_{3}$ are the weighting coefficient of the image local information parameter $M, S$, $C$ and the sum of the three is 1 . Since the value of $M, S, C$ represents different characteristics of the image, the selected value of $k_{1}, k_{2}, k_{3}$ can be determined according to its influence degree. After many experiments, it is found that the roughness compared with the other two local information parameters, the influence is greater, so takes $k_{3}=0.6$, the influence of image entropy is second, $k_{1}=0.3$, the influence of gradient amplitude is the least, and $k_{2}=0.1$.

The parameter $P=\lambda\left(k_{1} \cdot M+k_{2} \cdot S+k_{3} \cdot C\right), \lambda$ is adjustment parameter. Since the $E$ exponent is an increasing function in the real number domain, the enhancement range increases with the increase of the variable, which is in line with the purpose of the algorithm in the paper, so the fractional differential adaptive algorithm can be obtained,

$$
v=e^{P-Q}
$$

where $Q$ is the adjustment parameter. After many experiments with different images, it is found that the image enhancement effect is the most obvious when $\lambda=1, Q=1.7$.

\section{Simulation Experiment}

In this section, the segmentation algorithm proposed in the paper is first evaluated with two images. Then, the enhancement algorithm is analyzed with five images, and compared with the $[14,17,19]$. Finally, a numerical analysis of the experimental results is carried out.

Taking Lena image as an example, the effectiveness of the image segmentation algorithm in this paper is analyzed. Figure $4 \mathrm{~b}-\mathrm{d}$ are Lena images processed by three image segmentation algorithms, in which some local area is enlarged and observed. It can be seen that Figure 4e, $\mathrm{q}$ belong to the smooth area of the image, Figure $4 \mathrm{~m}$ belongs to the edge of the image, and Figure $4 i$ belongs to the texture area of the image. It can be seen from Figure $4 \mathrm{j}$ that the effect of rough set segmentation method on the processing of the image texture area is satisfactory, but the segmentation effect of the image smooth areas (Figure $4 \mathrm{f}, \mathrm{r}$ ) and edge of the image (Figure $4 \mathrm{n}$ ) is relatively poor. The image segmented by the particle swarm optimization method shows a excellent segmentation effect at the edge of the image (Figure 4o) and the smooth area of the image (Figure $4 \mathrm{~g}$, s), but the segmentation effect of the image texture area Figure $4 \mathrm{k}$ is poor. Finally, after combining the segmentation effects of the two methods, it can be seen that whether it is the smooth area Figure $4 \mathrm{~h}, \mathrm{t}$ of the image, or the edge Figure $4 \mathrm{p}$ and texture area Figure $4 \mathrm{i}$ of the image, the segmentation is relatively accurate.

The effectiveness of this algorithm is analyzed by the segmentation effect of plane image. Figure $5 b-d$ are plane images processed by three segmentation algorithms, and the same method is used to zoom in and observe some local areas. It can be seen that Figure $5 \mathrm{~m}, \mathrm{q}$ belong to the texture area of the image, Figure $5 \mathrm{i}$ belongs to the smooth area of the image, and Figure 5e belongs to edge of the image. From the enlarged images of Figure $5 n, r$, it can be seen that the segmentation effect of the mountains and the figures at the tail of the aircraft (texture area of the image) after the rough set method is better, while for the smooth area Figure 5j of the image and the edge of the image Figure 5f, the segmentation effect is poor. From the enlarged images of Figure $5 g, k$, it can be seen that the PSO method effectively segments both the smooth area and the edge of the image, while for the texture area of the image Figure 5o,s, the segmentation effect is not satisfactory. After combining the segmentation effect of the two methods, the segmentation effect of the rough set method on the texture is retained, and the PSO method is used to make up for 
the insufficient segmentation of the image edge and smooth area by the rough set method. A satisfactory segmentation effect is achieved.

Visual perception is used to evaluate the effectiveness of this enhancement algorithm.

Figure 7 is the experimental result of the Lena image. The enhancement effect of 0.4-order and 0.6-order are obvious, but the problem of excessive enhancement will occur in same local area. Ref. [17] enhances the image to a certain extent, but the effect is not obvious. Ref. [19] enhances edge and texture details but destroys some smooth areas. It can be seen that the enhancement effect of the image processed by 1-order is not obvious compared with other methods. Compared with the original image, the algorithm in the paper has obvious enhancement effect in the texture details such as eyes and hair. The enhancement effect is also satisfactory at the brim and the outline edge of the entire hat. There is almost no enhancement in the smooth area at the background and shoulders. Preserving the properties of the smooth area well. Compared with other algorithms, there is no problem of over-enhancing and destroying the smooth area, which illustrates the validity of the algorithm in the paper.

Figure 8 is the experimental result of the plane image. It can be seen that the letters on the plane in the original picture are relatively blurred, and the junction between the edge of the plane and the cloud layer is not obvious enough. The 0.6-order and 0.4-order has obvious over-enhancement problems in the enhancement of letters. It can be seen that the image of 1-order processing result is not very clear, because integer order processing will blur the image. The overall enhancement effect of [17] is not obvious. The overall enhancement effect of [19] is better but destroys the smooth area at the cloud layer. The processing results of the algorithm in the paper are the clearest letters, the processing results of mountains are also obvious better than other algorithms. In the paper, the contour part of the cloud layer is obviously enhanced, and the main part of the cloud layer is basically consistent with the original image, which once again shows that the algorithm in the paper has achieved a great balance between the enhancement of the texture area and the preservation of the smooth area.

For the processing results of Figure 9, the edge of the house of the processing results in the paper is more obvious, and the middle street part and the overall outline of the tree are also clearly visible. For the smooth area at the road, there is almost no major change. For the processing results of the Figure 10, the algorithm in the paper shows that the outlines of the three cars are very clear, and they are brighter than the original image after being enhanced in the grass, especially the texture on the front cover of the car below is very clear. The smooth area is almost unchanged, which achieves the effect of enhancing only the edge and texture details. For the aerial Figure 11, the entire sea surface belongs to the smooth area, after processing by the algorithm in the paper, this part is almost unchanged, while the texture details at the island are rich, which has a good enhancement effect on the edge contour. To sum up, the enhancement effect of the algorithm in the paper is clearer at the edge than other algorithms. The texture area has a better enhancement effect, and the smooth area is well maintained, which illustrates the validity of the algorithm in the paper again.

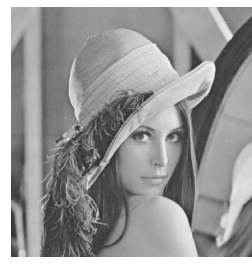

(a) (b)

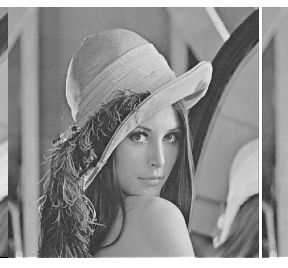

(c)

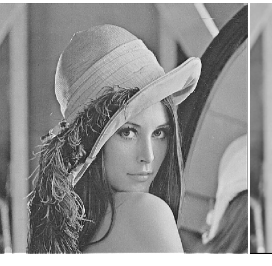

(d)

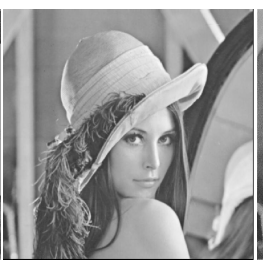

(e)

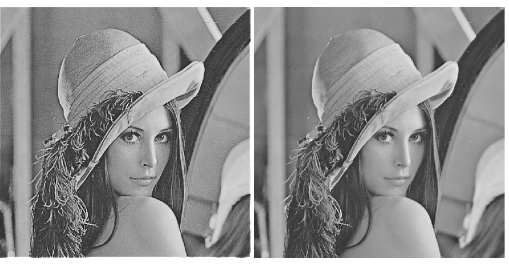

(f) $(\mathrm{g})$

Figure 7. The renderings of Lena image under different algorithms. (a) Original image. (b) 1-order. (c) 0.4-order. (d) 0.6-order. (e) [17]. (f) [19]. (g) Ours. 


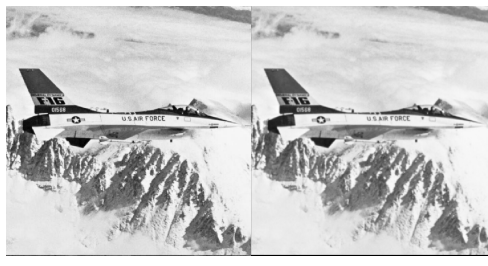

(a) (b)

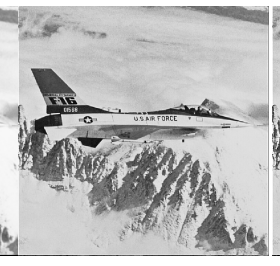

(c)

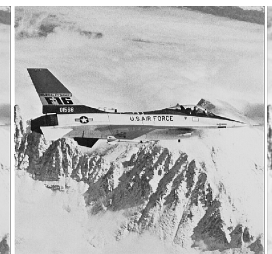

(d)

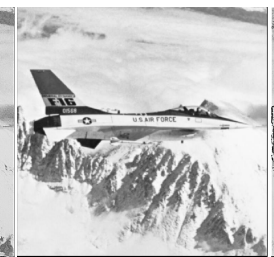

(e)

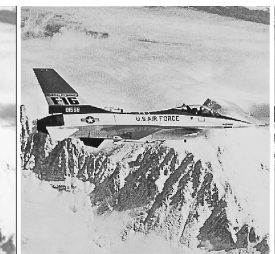

(f)

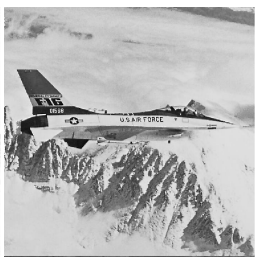

(g)

Figure 8. The renderings of plane image under different algorithms. (a) Original image. (b) 1-order. (c) 0.4-order. (d) 0.6-order. (e) [17]. (f) [19]. (g) Ours.

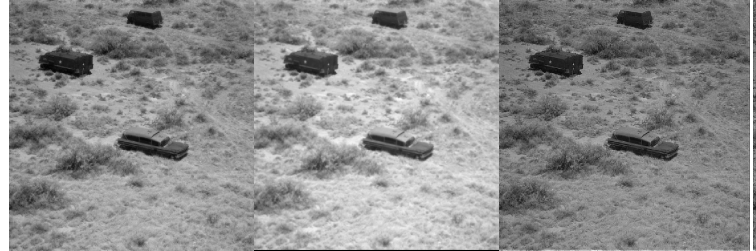

(a)

(b)

(c)

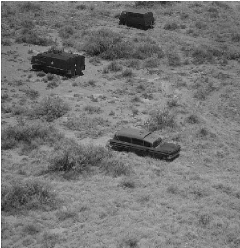

(d)

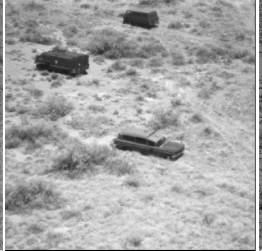

(e)

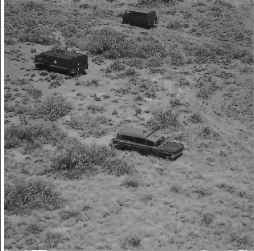

(f)

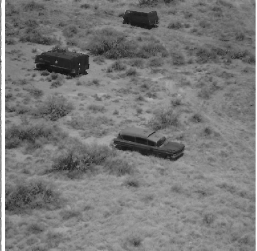

(g)

Figure 9. The renderings of trucks image under different algorithms. (a) Original image. (b) 1-order. (c) 0.4-order. (d) 0.6-order. (e) [17]. (f) [19]. (g) Ours.

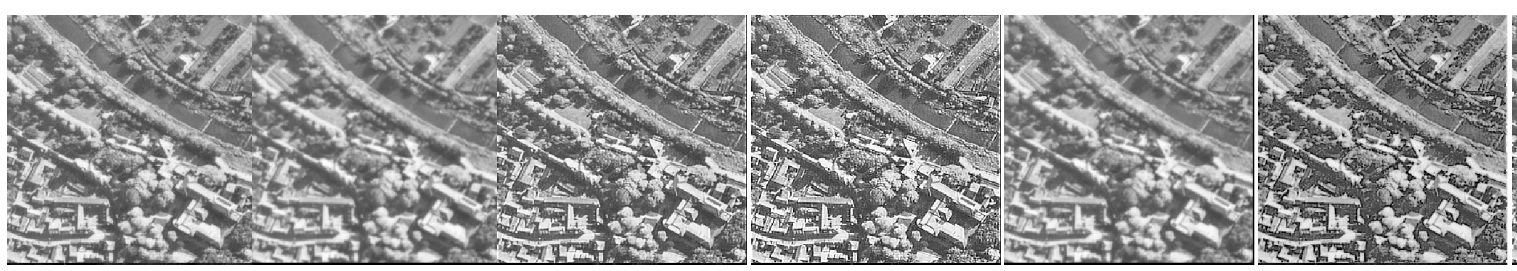

(a) (c) (d) (e)

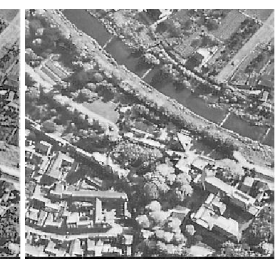

(g)

Figure 10. The renderings of street image under different algorithms. (a) Original image. (b) 1-order. (c) 0.4-order. (d) 0.6-order. (e) [17]. (f) [19]. (g) Ours.

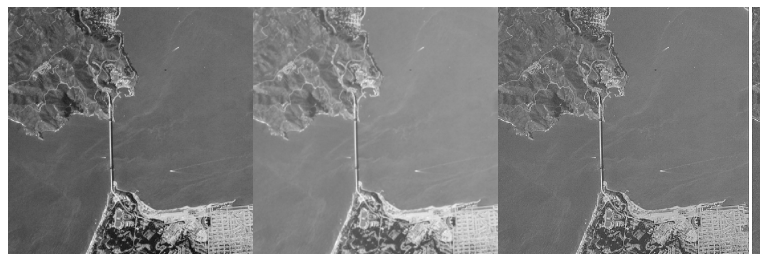

(a) (b)

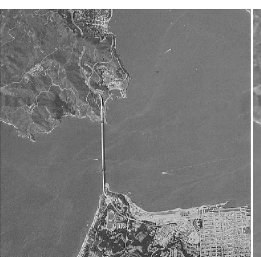

(d)

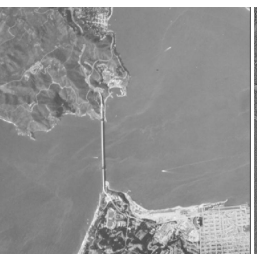

(e)

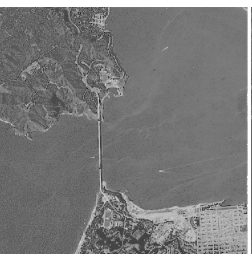

(f)

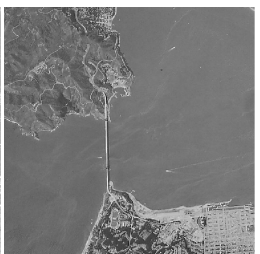

(g)

Figure 11. The renderings of aerial image under different algorithms. (a) Original image. (b) 1-order. (c) 0.4-order. (d) 0.6-order. (e) [17]. (f) [19]. (g) Ours.

In the paper, using SSIM [39] to detect the similarity of two images. The quality of the image can be detected by calculating the similarity of the structural information of the two images, and the visual features are better than the traditional MSE, the average Gradient and other methods are more in line with human visual perception. The value domain of SSIM is $[0,1]$, the value is larger, the image distortion is smaller, that is, the better the image visual effect. Its calculation formula is as follows, 


$$
\operatorname{SSIM}(x, y)=\frac{\left(2 \mu_{x} \mu_{y}+c_{1}\right)\left(2 \sigma_{x y}+c_{2}\right)}{\left(\mu_{x}^{2}+\mu_{y}^{2}+c_{1}\right)\left(\sigma_{x}^{2}+\sigma_{y}^{2}+c_{2}\right)}
$$

where: $\sigma_{x y}$ is the covariance of $x$ and $y ; \sigma_{x}$ is the variance of $x ; \mu_{x}$ is the mean value of $x ; \mu_{y}$ is the mean value of $y ; \sigma_{y}$ is the variance of $y ; c_{1}=\left(k_{1} L\right)^{2} ; c_{2}=\left(k_{2} L\right)^{2}$ are two variables that maintain stability; $L$ is the dynamic range of the pixel, and has a default value of $k_{1}=0.01, k_{2}=0.01$.

PSNR is the most widely used metric to evaluate the effect of image enhancement [40]. The larger the PSNR value, the less distortion and the better the image enhancement effect. Its calculation formula is as follows,

$$
\operatorname{PSNR}=10 \log _{10}\left[d^{2} / M S E\right],
$$

among them, $d$ is the maximum of the image gray value, MSE is the mean square error. $M S E$ is defined as follows,

$$
M S E=\frac{1}{x \times y} \sum_{i=1}^{x} \sum_{j=1}^{y}\|I(i, j)-O(i, j)\|^{2},
$$

where $(x, y)$ is the size of the image, $I(i, j)$ is the original image, $O(i, j)$ is the enhanced image.

Contrast is the degree of brightness and darkness of an image [41], which can reflect the clarity of the image. The greater the contrast, the clearer the visual effect of the image. The calculation formula of contrast is as follows,

$$
\text { Con }=\sum_{\delta} \delta(i, j)^{2} P_{\delta}(i, j),
$$

where $\delta(i, j)=|i-j|$ is the gray difference between adjacent pixels, and $P_{\delta}(i, j)$ represents the pixel distribution probability that the gray difference between adjacent pixels is $\delta$.

Gray level co-occurrence matrix is a matrix function of pixel distance and angle. It reflects the comprehensive information of image in direction, interval, change amplitude and speed by calculating the correlation between two gray levels in a certain distance and direction in the image. Because the amount of data of gray level co-occurrence matrix is usually large, it generally does not operate on the matrix directly, but extracts some of its feature quantities to describe the feature information of the original image, such as entropy, homogeneity, uniformity. The paper uses the statistical feature of homogeneity to evaluate the effect of image texture enhancement. Homogeneity is used to measure the uniformity of image texture distribution [35]. The greater the homogeneity, the more uniform the image texture area and the slower the change, indicating that the better the image enhancement effect. The calculation formula of homogeneity is as follows,

$$
H=\sum_{i, j} \frac{p(i, j)}{1+|i-j|}
$$

where $p(i, j)$ is the probability for gray-scale $i$ and $j$ and occurs at two pixels.

Therefore, SSIM represents the degree of smooth area retention, and PSNR represents the degree of edge detail and contour enhancement. Contrast reflects the clarity of the image, and homogeneity reflects the uniformity of the image texture area. It can be seen that from Tables 1 and 2, compared with other algorithms, the SSIM value of the algorithm in the paper is relatively large, and the PSNR value is relatively high, which indicates that the algorithm in the paper strengthens the edge and texture of the image while keeping the smooth area consistent with the original image as much as possible. However, the PSNR value of the image processed by the algorithm of 0.6 -order is relatively small, because the same fractional order processing the whole image will cause excessive enhancement. The enhancement also destroys the smooth area. Ref. [17] also has good results, but it is still insufficient compared with the algorithm in the paper. The enhancement algorithm 
proposed in [19] has a lower SSIM value because the smooth region is destroyed while enhancing. It can be seen from Table 3 that the contrast of the algorithm in the paper is slightly lower than other algorithms, because the proportion of the smooth area of the image is relatively large, and one of the main characteristics of the algorithm in the paper is to retain the characteristics of the smooth area, so the contrast of the image processed by the algorithm in the paper is not the highest. It can be seen from Table 4 that the homogeneity of the images processed by these algorithms is not much different, but the homogeneity of the five images processed by the algorithm in the paper is relatively large, which shows that the enhancement effect of the algorithm in the paper is very suitable for all kinds of images. It can be seen from the above analysis that the algorithm in the paper enhances the image edge and texture details and retain the information of the smooth area of the image optimally, which also reflects the effectiveness of the algorithm in the paper.

Table 1. Under different algorithms, the Structural Similarity Index Measure (SSIM) values of different images.

\begin{tabular}{ccccccc}
\hline & Ours & $\mathbf{0 . 4 - O r d e r}$ & $\mathbf{0 . 6 - O r d e r}$ & 1-Order & [17] & [19] \\
\hline Lena & $\mathbf{0 . 9 5 4 0}$ & 0.9076 & 0.7012 & 0.9260 & 0.9332 & 0.6700 \\
plane & $\mathbf{0 . 9 5 6 6}$ & 0.9269 & 0.7647 & 0.9470 & 0.9526 & 0.7529 \\
Trucks & $\mathbf{0 . 9 7 6 1}$ & 0.8802 & 0.6299 & 0.9030 & 0.9154 & 0.7869 \\
Street & 0.8478 & 0.8812 & 0.6726 & 0.8870 & $\mathbf{0 . 9 0 1 7}$ & 0.7782 \\
Aerial & $\mathbf{0 . 9 2 0 8}$ & 0.8829 & 0.6223 & 0.9033 & 0.9156 & 0.7550 \\
\hline
\end{tabular}

Table 2. Under different algorithms, the PSNR values of different images.

\begin{tabular}{ccccccc}
\hline & Ours & 0.4-Order & 0.6-Order & 1-Order & [17] & [19] \\
\hline Lena & $\mathbf{3 0 . 7 1 1}$ & 30.6453 & 24.3025 & 26.1858 & 26.2479 & 21.8462 \\
plane & $\mathbf{2 8 . 5 7 2}$ & 27.3842 & 23.9278 & 25.2817 & 25.3700 & 20.5966 \\
Trucks & $\mathbf{3 6 . 2 7 4}$ & 30.9549 & 23.6656 & 27.0064 & 27.0836 & 13.2124 \\
Street & 21.974 & $\mathbf{2 3 . 9 3 5 7}$ & 17.7327 & 23.1423 & 16.3556 & 19.5258 \\
Aerial & 26.887 & $\mathbf{2 8 . 6 4 3 1}$ & 21.6291 & 26.8077 & 25.9583 & 24.7166 \\
\hline
\end{tabular}

Table 3. Under different algorithms, the contrast values of different images.

\begin{tabular}{ccccccc}
\hline & Ours & 0.4-Order & 0.6-Order & 1-Order & [17] & [19] \\
\hline Lena & 34.9285 & 35.3760 & $\mathbf{3 6 . 7 4 8 6}$ & 33.3108 & 33.3612 & 35.6323 \\
plane & 34.7625 & 35.5782 & 37.5650 & 32.8419 & 32.9148 & $\mathbf{3 9 . 6 3 6 9}$ \\
Trucks & 20.2284 & 21.0476 & $\mathbf{2 4 . 5 9 5 9}$ & 18.2502 & 18.3030 & 23.2832 \\
Street & 45.6793 & 45.8204 & $\mathbf{5 5 . 0 1 2 0}$ & 33.4451 & 33.7412 & 47.1636 \\
Aerial & 15.4079 & 15.8663 & $\mathbf{2 1 . 0 3 0 2}$ & 11.8307 & 11.9199 & 15.3956 \\
\hline
\end{tabular}

Table 4. Under different algorithms, the homogeneity values of different images.

\begin{tabular}{ccccccc}
\hline & Ours & $\mathbf{0 . 4 - O r d e r}$ & $\mathbf{0 . 6}-$ Order & 1-Order & [17] & [19] \\
\hline Lena & 0.9997 & $\mathbf{0 . 9 9 9 9}$ & 0.9970 & 0.9966 & 0.9966 & 0.9961 \\
plane & 0.9982 & $\mathbf{0 . 9 9 9 7}$ & 0.9963 & 0.9966 & 0.9966 & 0.9936 \\
Trucks & $\mathbf{0 . 9 9 9 9}$ & 0.9998 & 0.9984 & 0.9956 & 0.9966 & 0.9989 \\
Street & $\mathbf{0 . 9 9 9 4}$ & 0.9994 & 0.9947 & 0.9932 & 0.9932 & 0.9674 \\
Aerial & $\mathbf{0 . 9 9 8 1}$ & 0.9980 & 0.9932 & 0.9966 & 0.9966 & 0.9957 \\
\hline
\end{tabular}

It can be seen from the above analysis that the main advantage of the algorithm in the paper is that it can effectively enhance the image edge and texture area while retaining the characteristics of the smooth area of the image. This is because the algorithm in the paper combines the advantages of rough set and PSO, accurately segments the smooth area, edge and texture area of the image. According to the local characteristics of the image, an adaptive image enhancement algorithm is designed to enhance the edge and texture area 
obtained after image segmentation. At the same time, the smooth area of the image is not enhanced, so that the characteristics of the smooth area of the image can be well preserved.

\section{Conclusions}

In the paper, two image segmentation methods are firstly proposed. The segmentation algorithm based on rough set better segments the texture area of the image, and the segmentation algorithm based on PSO effectively segments the smooth area and edge part of the image. Combining the advantages of two kinds of image segmentation, the image is accurately divided into smooth area, edge and texture area. According to the image segmentation results, the adaptive fractional differential filter is used to effectively enhance the image. Experiments show that the algorithm in the paper well solves the problem of excessive enhancement and destruction of smooth areas in image enhancement.

However, the method proposed in the paper still has shortcomings in practical application. In the future research work, we need to focus on solving the following problems: how we can more accurately determine the relationship between the local information of the image and the fractional differential order, so as to construct a more accurate adaptive function; and how we can further reduce the time required to process the image by optimizing the algorithm.

Author Contributions: These authors contributed equally to this work. Conceptualization, methodology, software, validation, X.Z., R.L., J.R. and Q.G.; formal analysis, J.R.; investigation, Q.G.; resources, J.R.; data curation, Q.G.; writing—original draft preparation, Q.G.; writing—review and editing, X.Z.; visualization, J.R.; supervision, Q.G.; project administration, X.Z. and J.R.; funding acquisition, X.Z. All authors have read and agreed to the published version of the manuscript.

Funding: National key research and development program topic (2020YFB1710003).

Institutional Review Board Statement: Not applicable.

Informed Consent Statement: Not applicable.

Data Availability Statement: Not applicable.

Conflicts of Interest: The authors declare no confilct of interest.

\section{References}

1. Oktay, O. Application to cardiac image enhancement and segmentation. IEEE Trans. Med. Imaging 2018, 37, 384-395. [CrossRef]

2. Chen, C.; Qi, L.; Jun, Z. Retinal image enhancement using low-pass filtering and $\alpha$-rooting. Signal Process. 2020, $170,107445$.

3. Dong, T.; Jian, W.; Feng, D.; Wen, L.; Xiao, H.; Yu, Z.; Tong, T.; Sheng, M.; Dong, C.; Ming, L. Medical imaging and diagnosis of subpatellar vertebrae based on improved laplacian image inhancement algorithm. Comput. Methods Programs Biomed. 2020, 187, 105082.

4. Wen, W.; Mao, W.; Qiu, J.; Ru, W.; Hong, G. L(1/2) Regularization for ISAR imaging and target enhancement of complex image. IEEE Trans. Geosci. Remote Sens. 2022, 60, 1-10. [CrossRef]

5. Fei, P. UW-GAN: Single-image depth estimation and image enhancement for underwater images. IEEE Trans. Instrum. Meas. 2021, 70, 1-12.

6. Khan, N.A.; Sandsten, M. Time-frequency image enhancement based on interference suppression in wigner-ville distribution. Signal Process. 2016, 127, 80-85. [CrossRef]

7. Jie, G.; Yu, L.; Bin, L. LIME: Low-light image enhancement via illumination map estimation. IEEE Trans. Image Process. 2010, 26, 982-993.

8. Lee, E.; Kim, S.; Kang, W.; Seo, D.; Paik, J. Contrast enhancement using dominant brightness level analysis and adaptive intensity transformation for remote sensing images. IEEE Geosci. Remote Sens. Lett. 2013, 10, 62-66. [CrossRef]

9. Iqbal, M.Z.; Ghafoor, A.; Siddiqui, A.M. Satellite image resolution enhancement using dual-tree complex wavelet transform and nonlocal means. IEEE Geosci. Remote Sens. Lett. 2013, 10, 451-455. [CrossRef]

10. Pyka, K. Wavelet-based local contrast enhancement for satellite, aerial and close range images. Remote Sens. 2017, 9, 25. [CrossRef]

11. Fei, P. Fractional-order Euler-Lagrange equation for fractional-order variational method: A necessary condition for fractional-order fixed boundary optimization problems in signal processing and image processing. IEEE Access 2016, 4, 10110-10135.

12. Li, C.; Quan, C.; Yu, X. Digital fractional order Savitzky-Golay differentiator. IEEE Trans. Circuits Syst. II Express Briefs 2011, 58, 758-762.

13. Saadia, A.; Rashdi, A.R. Incorporating fractional calculus in echo-cardiographic image denoising. Comput. Electr. Eng. 2018, 67, 134-144. [CrossRef] 
14. Fei, P.; Liu, Z.; Xiao, Y. Fractional differential mask: A fractional differential-based approach for multiscale texture enhancement. IEEE Trans. Image Process. 2010, 19, 491-511.

15. Zi, L.; Yi, T.; Bing, J.; Hirasawa, K. Adaptive fractional-order totalvariation image restoration with split Bregman iteration. ISA Trans. 2017, 82, 210-222.

16. Fei, P.; Liu, Z.; Siarry, P.; Ni, Z.; Guang, L. Fractional partial differential equation: Fractional total variation and fractional steepest descent approach-based multiscale denoising model for texture image. Abstr. Appl. Anal. 2013, 2013, 483791.

17. Li, C.; Quan, C.; Yu, X. 1-D and 2-D digital fractional-order Savitzky-Golay differentiator. Image Video Process. $2012,6,503-511$.

18. Bo, L.; Wei, X. Adaptive fractional differential approach and its application to medical image enhancement. Comput. Electr. Eng. 2015, 45, 324-335.

19. Feng, Z.; Hui, Y. Image enhancement algorithm using adaptive fractional differential mask technique. Math. Found. Comput. 2019, 2, 347-359.

20. Dai, S. Rough approximation operators on a complete orthomodular lattice. Axioms 2021, 10, 164. [CrossRef]

21. Pawlak, Z. Rough sets. Int. J. Comput. Inf. Sci. 1982, 11, 341-356. [CrossRef]

22. Pawlak, Z. Rough set theory and its applications. J. Telecommun. Inf. Technol 2002, 3, 7-10.

23. Phophalia, A.; Mitra, S.K. 3D MR image denoising using rough set and kernel PCA method. Magn. Reson. Imaging 2016, 36, 135-145. [CrossRef]

24. Xuan, J.; Bo, H.; Yong, X.; Hui, Z. A robust modified gaussian mixture model with rough set for image segmentation. Neurocomputing 2017, 266, 550-565.

25. Namburu, A.; Samay, S.K.; Edara, S.R. Soft fuzzy rough set-based MR brain image segmentation. Appl. Soft Comput. 2017, 54, 456-466. [CrossRef]

26. Guo, X.; Wang, Y. Multi-objective model for logistics sistribution programming considering logistics service level. Southwest Jiaotong Univ. 2012, 47, 874-880.

27. Pham, T.X.; Siarry, P.; Oulhadj, H. Integrating fuzzy entropy clustering with an improved PSO for MRI brain image segmentation Appl. Soft Comput. 2018, 65, 230-242. [CrossRef]

28. Borjigin, S.; Sahoo, P.K. Color image segmentation based on multi-level tsallis-havrda-charvát entropy and 2D histogram ssing PSO algorithms. Pattern Recognit. 2019, 92, 107-118. [CrossRef]

29. Feng, Z.; Kai, H. Adaptive neural network sliding mode control for nonlinear singular fractional order aystems with mismatched uncertainties. Fractal Fract. 2020, 4, 50.

30. Chang, L.; Feng, Z. Adaptive sliding mode observer design for a class of T-S fuzzy descriptor fractional order systems. IEEE Trans. Fuzzy Syst. 2020, 28, 1951-1959.

31. Zhang, X.F.; Dong, J. LMI criteria for admissibility and robust stabilization of singular fractional-order systems possessing poly-topic uncertainties. Fractal Fract. 2020, 4, 58. [CrossRef]

32. Zhang, X.F.; Yan, Y.Q. Admissibility of fractional order descriptor systems based on complex variables: An LMI approach. Fractal Fract. 2020, 4, 8. [CrossRef]

33. Zhang, X.F.; Chen, Y.Q. Admissibility and robust stabilization of continuous linear singular fractional order systems with the fractional order $\alpha$ : The $0<\alpha<1$ case. ISA Trans. 2017, 82, 42-50. [PubMed]

34. Candan, C.; Kutay, M.A.; Ozaktas, H.M. The discrete fractional Fourier transform. IEEE Trans. Signal Process. 2000, $48,1329-1337$. [CrossRef]

35. Jalab, H.A.; Ibrahim, R.W. Texture enhancement based on the Savitzky-Golay fractional differential operator. Math. Probl. Eng. 2013, 2013, 149289. [CrossRef]

36. Gao, C.B.; Zhou, J.L.; Hu, J.R.; Lang, F.N. Edge detection of colour image based on quaternion fractional differential. IET Image Process 2011, 5, 261-272. [CrossRef]

37. Bo, L.; Wei, X. Adaptive fractional differential algorithm based on Otsu standard. In Proceedings of the 26th Chinese Control and Decision Conference (2014 CCDC), Changsha, China, 31 May-2 June 2014; pp. 2021-2026.

38. Kumar, R.; Kulashekar, P.; Dhanasekar, B.; Ramamoorthy, B. Application of digital image magnification for surface roughness evaluation using machine vision. Int. J. Mach. Tool. Manu 2005, 45, 228-234. [CrossRef]

39. Xu, K.; Chen, H.; Xu, C.; Jin, Y.; Zhu, C. Structure-Texture aware network for low-light image enhancement. IEEE Trans. Circuits Syst. Video Technol. 2022, 10, 1109. [CrossRef]

40. Lecca, M. STAR: A segmentation-based approximation of point-based sampling milano retinex for color image enhancement. IEEE Trans. Image Process. 2018, 27, 802-812. [CrossRef]

41. Khan, M.F.; Khan, E.; Abbasi, Z.A. Segment selective dynamic histogram equalization for brightness preserving contrast enhancement of images. Optik 2014, 125, 1385-1389. [CrossRef] 